\title{
Defect Corrections for Multigrid Solutions of the Dirichlet Problem in General Domains
}

\author{
By Winfried Auzinger*
}

\begin{abstract}
Recently, the technique of defect correction for the refinement of discrete solutions to elliptic boundary value problems has gained new acceptance in connection with the multigrid approach. In the present paper we give an analysis of a specific application, namely to finite-difference analogues of the Dirichlet problem for Helmholtz's equation, emphasizing the case of nonrectangular domains. A quantitative convergence proof is presented for a class of convex polygonal domains.
\end{abstract}

1. Introduction. The purpose of this paper is to study the behavior of a defect correction method for the linear elliptic boundary value problem

$$
\begin{aligned}
-\Delta u(x, y)+c(x, y) u(x, y) & =f(x, y), & & (x, y) \in \Omega, \\
u(x, y) & =g(x, y), & (x, y) & \in \partial \Omega,
\end{aligned}
$$

in a general domain $\Omega \subset \mathbf{R}^{2}$. The method and, in particular, its combination with the multigrid approach, has been discussed by Auzinger and Stetter [4] and Hackbusch [10]. (We also refer to the work of Frank, Hertling, and Monnet [7].)

Defect correction is a way to obtain, in an iterative or semi-iterative fashion, solutions to complex problems by means of solving related, simpler problems. In the present application, the "complex problem" is a high-order discretization, whereas the "simpler problem" is a low-order scheme which is solved by standard multigrid. It is our aim to show, in a concrete nontrivial situation, that this is a reasonable way to get a high-order solution. More precisely, we prove that contraction rates can be obtained which yield the usual multigrid efficiency. Our approach has the advantage that standard multigrid software can be used for the solution phase in a black box manner. The high-order scheme is only involved in an outer iteration. Thus, high accuracy is introduced in such a way as to be (theoretically and computationally) clearly separated from the inversion process.

After some general remarks in Section 2, we present in Section 3 an account of the model problem analysis given in Auzinger [1]. In Section 4, which is the heart of the paper, we derive explicit bounds for the contraction number of the defect correction iteration for a class of convex polygonal domains. That section includes a quantitative $\mathrm{H}^{2}$-regularity estimate for the discrete Poisson equation. Our analysis does not

Received May 30, 1985; revised March 4, 1986.

1980 Mathematics Subject Classification. Primary 65B05, 65N20.

*This work was supported by "Österreichischer Fonds zur Förderung der wissenschaftlichen Forschung".

(C)1987 American Mathematical Society $0025-5718 / 87 \$ 1.00+\$ .25$ per page 
depend on asymptotic error expansions. Some remarks on general domains can be found in Section 5.

Throughout we have adopted the convention of "generic constants" $C, C_{1}, \ldots$ These are always independent of the discretization level.

2. Basic Properties. Let

$$
L u=f
$$

denote a linear elliptic boundary value problem of second order (e.g., (1.1)) and

$$
L_{h} u_{h}=f_{h}
$$

its discretization on a grid with mesh size $h$. (2.2) is assumed to be stable in some norm $\|\cdot\|$ and will be referred to as the "basic discretization" of (2.1). In our application, (2.2) is a 2 nd-order method.

Let, in addition,

$$
L_{h}^{\prime} u_{h}=f_{h}^{\prime}
$$

be a discretization of higher order. Within our defect correction approach, (2.3) will not be inverted but evaluated: Given a discrete approximation $u_{h}^{(i)}$, its defect with respect to (2.3) defines a corrected version of (2.2) in the following way:

$$
L_{h} u_{h}^{(i+1)}=L_{h} u_{h}^{(i)}-\left(L_{h}^{\prime} u_{h}^{(i)}-f_{h}^{\prime}\right) .
$$

This yields an iteration procedure possessing any solution of (2.3) as a fixed point. Within this context, (2.3) will be referred to as the "target discretization" of (2.1). (See Stetter [12] for the general principle of defect correction.)

With the notation

$$
\Delta L_{h}:=L_{h}-L_{h}^{\prime},
$$

the iteration (2.4) reads

$$
u_{h}^{(i+1)}:=L_{h}^{-1} \Delta L_{h} u_{h}^{(i)}+L_{h}^{-1} f_{h}^{\prime} .
$$

The general structure of our analysis of the defect correction iteration (2.4) can be described as follows: Let $u_{h}^{*}$ be the restriction of the true solution $u^{*}$ of (2.1) to the grid with mesh size $h$. For the error function

$$
e_{h}^{(i)}:=u_{h}^{(i)}-u_{h}^{*}
$$

we obtain the "error iteration" equivalent to (2.4):

$$
e_{h}^{(i+1)}:=L_{h}^{-1} \Delta L_{h} e_{h}^{(i)}-L_{h}^{-1}\left(L_{h}^{\prime} u_{h}^{*}-f_{h}^{\prime}\right) .
$$

Thus, we will have to investigate

(i) the contraction behavior of $L_{h}^{-1} \Delta L_{h}$,

(ii) the role of the inhomogeneous term $L_{h}^{-1}\left(L_{h}^{\prime} u_{h}^{*}-f_{h}^{\prime}\right)$.

We have not presupposed stability of (2.3). In fact, for the purpose of defect correction, unstable target discretizations are usually admitted. It must, however, be pointed out that the "global" contractivity of the defect correction operator $L_{h}^{-1} \Delta L_{h}$ and the stability of (2.3) are directly interrelated: $L_{h}^{-1} \Delta L_{h}$ cannot be contractive, independent of $h$, if $L_{h}^{\prime}$ is unstable or even fails to be invertible. (See, however, our remarks at the end of this section. See also Section 5 for further comments on unstable target discretizations.) 
Proposition 2.1. Let $L_{h}$ be stable and $L_{h}^{-1} \Delta L_{h}$ have a contraction number $\left\|L_{h}^{-1} \Delta L_{h}\right\| \leqslant k<1$ independent of $h$. Then $L_{h}^{\prime}$ is invertible and stable:

$$
\left\|L_{h}^{\prime-1}\right\| \leqslant \frac{1}{1-k}\left\|L_{h}^{-1}\right\|
$$

For the error $e_{h}^{\prime}:=u_{h}^{\prime}-u_{h}^{*}$ (i.e., the fixed point of (2.6)) we obtain

$$
\left\|e_{h}^{\prime}\right\| \leqslant \frac{1}{1-k}\left\|L_{h}^{-1}\left(L_{h}^{\prime} u_{h}^{*}-f_{h}^{\prime}\right)\right\|
$$

Proof. Since $\left\|I_{h}-L_{h}^{-1} L_{h}^{\prime}\right\|<1, L_{h}^{-1} L_{h}^{\prime}$ is invertible, and so is $L_{h}^{\prime}$. Moreover,

$$
\left\|L_{h}^{\prime-1}\right\| \leqslant\left\|L_{h}^{-1}\right\|+\left\|\left(I_{h}-L_{h}^{-1} L_{h}^{\prime}\right) L_{h}^{\prime-1}\right\| \leqslant\left\|L_{h}^{-1}\right\|+k\left\|L_{h}^{\prime-1}\right\|,
$$

from which we infer (2.7). A similar argument establishes (2.8)

For an extension of this simple result, see Proposition 2.2 below.

The convergence behavior of the defect correction iteration (2.4) is essentially retained if $L_{h}$ is only approximately inverted: Assume

$$
\left\|I_{h}-K_{h} L_{h}\right\| \leqslant l<1,
$$

independent of $h$. (This can be expected if $K_{h}$ represents a suitable multigrid cycle.) Then the iteration

$$
u_{h}^{(i+1)}:=\left(I_{h}-K_{h} L_{h}^{\prime}\right) u_{h}^{(i)}+K_{h} f_{h}^{\prime}
$$

has a contraction number $\leqslant \tilde{k}<1$ if

$$
\tilde{k}:=k+l+k l<1
$$

( $k$ from Proposition 2.1; cf. Auzinger and Stetter [4]).

Since, in our situation, the actual convergence rate depends crucially on the smoothness of the "algebraic error" (i.e., the error with respect to the fixed point), we shall also consider the following modification of (2.4) (or (2.10)):

$$
\bar{u}_{h}^{(i)}:=\left(I_{h}-T_{h} L_{h}\right) u_{h}^{(i)}+T_{h} f_{h}, \quad u_{h}^{(i+1)}:=L_{h}^{-1} \Delta L_{h} \bar{u}_{h}^{(i)}+L_{h}^{-1} f_{h}^{\prime}
$$

(or $K_{h}$ instead of $L_{h}^{-1}$, respectively). Here, the defect correction is "preconditioned" by a smoothing sweep relative to the basic discretization. We expect that a suitable multigrid smoother $T_{h}$ will improve the convergence considerably.

On the other hand, a fixed point $\hat{u}_{h}$ of (2.12) does no longer satisfy (2.3). In the following we give a representation of $\hat{u}_{h}$. (See also Hackbusch [10] for a related result.)

Proposition 2.2. Fixed-point shift. Let $M_{h}:=L_{h}^{-1} \Delta L_{h}\left(I_{h}-T_{h} L_{h}\right)$, and assume $\left\|M_{h}\right\| \leqslant m<1, m$ independent of $h$. Then there is a unique fixed point $\hat{u}_{h}$ of (2.12) satisfying

$$
\hat{L}_{h} \hat{u}_{h}=\hat{f}_{h}
$$

where $\hat{L}_{h}$ and $\hat{f}_{h}$ are defined by

$$
\hat{L}_{h}:=L_{h}^{\prime}+\Delta L_{h} T_{h} L_{h}, \quad \hat{f}_{h}:=f_{h}^{\prime}+\Delta L_{h} T_{h} f_{h} .
$$

$\hat{L}_{h}$ is invertible and stable with

$$
\left\|\hat{L}_{h}^{-1}\right\| \leqslant \frac{1}{1-m}\left\|L_{h}^{-1}\right\| .
$$


The error $\hat{e}_{h}:=\hat{u}_{h}-u_{h}^{*}$ is

$$
\hat{e}_{h}=-\left(I_{h}-M_{h}\right)^{-1} L_{h}^{-1}\left[\left(L_{h}^{\prime} u_{h}^{*}-f_{h}^{\prime}\right)+\Delta L_{h} T_{h}\left(L_{h} u_{h}^{*}-f_{h}\right)\right] .
$$

Proof. Combining the steps in (2.12), we obtain the fixed-point equation

$$
\hat{u}_{h}=M_{h} \hat{u}_{h}+L_{h}^{-1}\left[f_{h}^{\prime}+\Delta L_{h} T_{h} f_{h}\right] \text {, }
$$

hence, $L_{h}\left(I_{h}-M_{h}\right) \hat{u}_{h}=\hat{f}_{h}$. Moreover, $L_{h}\left(I_{h}-M_{h}\right)=\hat{L}_{h}$ as defined in (2.14), since

$$
\begin{aligned}
M_{h} & =\left(I_{h}-L_{h}^{-1} L_{h}^{\prime}\right)\left(I_{h}-T_{h} L_{h}\right) \\
& =I_{h}-L_{h}^{-1}\left(L_{h}^{\prime}+\Delta L_{h} T_{h} L_{h}\right)=I_{h}-L_{h}^{-1} \hat{L}_{h} .
\end{aligned}
$$

Together with $\left\|M_{h}\right\| \leqslant m<1$, this implies (2.15). The error $\hat{e}_{h}$ is given by

$$
\hat{e}_{h}=-\hat{L}_{h}^{-1}\left(\hat{L}_{h} u_{h}^{*}-\hat{f}_{h}\right) \text {, }
$$

which is easily seen to be equivalent to (2.16).

Proposition 2.2 shows that $\hat{L}_{h}$ is stable under weaker assumptions than $\left\|L_{h}^{-1} \Delta L_{h}\right\|$ $<1$. Hence it is possible that $\hat{L}_{h}$ is stable even if $L_{h}^{\prime}$ is not; $\hat{L}_{h}$ may be considered a "stabilization" of $L_{h}^{\prime}$. Although this observation has no immediate consequence in our application (cf. Sections 3 and 4), it may be useful in other cases.

Naturally, the modification (2.12) will only make sense if, within (2.16), the "perturbation" involving the low-order truncation error $L_{h} u_{h}^{*}-f_{h}$ does not destroy the accuracy controlled by $L_{h}^{-1}\left(L_{h}^{\prime} u_{h}^{*}-f_{h}^{\prime}\right)$. We shall return to this question in the sections which follow.

3. Model Problem Analysis. In this section we consider Helmholtz's equation in the unit square. This example has already been discussed in Auzinger and Stetter [4] and, in more deiail, in Auzinger [1]. Let $\Omega=(0,1) \times(0,1)$, and let Helmholtz's equation (1.1) be given. The quoted results apply to the case of $c \equiv$ const $>0$. (See [1] for the handling of variable $c(x, y)$ by partial summation.) On a uniform grid with mesh spacing $h=2^{-m}, m \in \mathbf{N}$, the basic discretization $L_{h} u_{h}=f_{h}$ is defined by the usual five-point stencil

$$
\frac{1}{h^{2}}\left[\begin{array}{rrr} 
& -1 & \\
-1 & 4 & -1 \\
& -1 &
\end{array}\right]
$$

and by straightforward point evaluation for $c u$ and $f . L_{h} u_{h}=f_{h}$ is a stable, 2nd-order discretization.

Let the target discretization $L_{h}^{\prime} u_{h}=f_{h}^{\prime}$ be given by the well-known stable, 4th-order "Mehrschrittverfahren". Then, $\Delta L_{h}=L_{h}-L_{h}^{\prime}$ is given by

$$
\Delta L_{h} u_{h} \sim \frac{1}{6 h^{2}}\left[\begin{array}{rrr}
1 & -2 & 1 \\
-2 & 4 & -2 \\
1 & -2 & 1
\end{array}\right] u_{h}+\frac{1}{12}\left[\begin{array}{rrr} 
& -1 & \\
-1 & 4 & -1 \\
& -1 &
\end{array}\right] c u_{h} \text {. }
$$

Now let $\|\cdot\|_{2}$ denote the (properly scaled) Euclidean norm as well as its associated operator norm. With respect to $\|\cdot\|_{2}, L_{h}^{-1} \Delta L_{h}$ has a contraction number independent of $h$ :

Proposition 3.1 .

$$
\left\|L_{h}^{-1} \Delta L_{h}\right\|_{2} \leqslant \frac{1}{3}+O\left(c h^{2}\right) \leqslant \frac{2}{3} .
$$

Proof. Given in [1]. 
Here and in the sequel, $O\left(c h^{2}\right)$ means $\psi(c) \cdot O\left(h^{2}\right)$, where $\psi(c)=O(c)$ for small $c$, but uniformly bounded for arbitrary $c$. In (3.3), the bound $\frac{2}{3}$ is valid independent of $h$ and $c$. The analysis of the modified iteration (2.12) is based on the following estimate.

LEMMA 3.2.

$$
\left\|L_{h}^{-1} \Delta L_{h} L_{h}^{-1}\right\|_{2} \leqslant \frac{h^{2}}{24}\left(1+O\left(c h^{2}\right)\right) \leqslant \frac{h^{2}}{6} .
$$

Proof. Given in [1].

Assume that, in (2.12), the smoothing step consists in $\nu \geqslant 1$ applications of an appropriate relaxation procedure, say

$$
I_{h}-T_{h} L_{h}=S_{h}^{\nu},
$$

and assume further that $S_{h}$ has the smoothing property (defined in Hackbusch [9]) for $\|\cdot\|_{2}$ and $\alpha=2$ :

$$
\left\|L_{h} S_{h}^{\nu}\right\|_{2} \leqslant C(\nu) h^{-2}
$$

with $C(\nu) \rightarrow 0$ as $\nu \rightarrow \infty$.

Proposition 3.3. Let $S_{h}$ satisfy (3.5). Then,

$$
\left\|L_{h}^{-1} \Delta L_{h} S_{h}^{\nu}\right\|_{2} \leqslant \frac{1}{24} C(\nu)\left(1+O\left(c h^{2}\right)\right) \leqslant \frac{1}{6} C(\nu)
$$

Proof. Use the splitting $L_{h}^{-1} \Delta L_{h} S_{h}^{\nu}=L_{h}^{-1} \Delta L_{h} L_{h}^{-1} L_{h} S_{h}^{\nu}$ and apply Lemma 3.2.

The smoothing property (3.5) has been proved in [9] for damped Jacobi and "red-black" Gauss/Seidel smoothers with

$$
\begin{aligned}
& C(\nu) \leqslant \frac{6}{2 \nu+1}\left(1+O\left(c h^{2}\right)\right), \\
& C(\nu) \leqslant \frac{8}{3 \sqrt{3} \nu}\left(1+O\left(c h^{2}\right)\right),
\end{aligned}
$$

respectively.

The following table shows the resulting bounds for $\left\|L_{h}^{-1} \Delta L_{h} S_{h}^{\nu}\right\|_{2}, \nu=1,2, \ldots$, in the case of Poisson's equation.

\begin{tabular}{c||c|c}
\hline$\nu$ & Jacobi & Gauss/Seidel \\
\hline \hline 1 & .083 & .064 \\
2 & .050 & .032 \\
3 & .036 & .021 \\
4 & .028 & .016 \\
\hline
\end{tabular}

These numbers are comparable in size to typical multigrid convergence factors.

We conclude this section by showing that the "fixed-point shift" (cf. Proposition 2.2 ) is $O\left(h^{4}\right)$. Hence, the smoother does not affect the order of accuracy.

Lemma 3.4. For $T_{h}=\left(I_{h}-S_{h}^{\nu}\right) L_{h}^{-1}$, there exists $C^{\prime}(\nu)$ independent of $h$ such that

$$
\left\|T_{h}\right\|_{2} \leqslant C^{\prime}(\nu) h^{2}
$$


This holds for both of the smoothing procedures considered. For Jacobi relaxation, $C^{\prime}(\nu) \leqslant C \cdot \nu ;$ for Gauss/Seidel, $C^{\prime}(\nu) \leqslant C \cdot\left(2^{\nu}-1\right)$.

Proof. See [2].

Proposition 3.5. Let $\left\|L_{h}^{-1} \Delta L_{h} S_{h}^{\nu}\right\|_{2} \leqslant m<1$. There exist constants $C_{1}, C_{2}$ independent of $h$ such that the error $\hat{e}_{h}$ of Proposition 2.2 satisfies

$$
\left\|\hat{e}_{h}\right\|_{2} \leqslant \frac{1}{1-m}\left[C_{1}\left\|L_{h}^{\prime} u_{h}^{*}-f_{h}^{\prime}\right\|_{2}+C_{2} C^{\prime}(\nu) h^{2}\left\|L_{h} u_{h}^{*}-f_{h}\right\|_{2}\right] .
$$

Thus, $\left\|\hat{e}_{h}\right\|_{2}=O\left(h^{4}\right)$.

Proof. Use the representation (2.16), (3.3), (3.8) and the stability of $L_{h}$.

4. Convergence Analysis for a Class of Convex Polygonal Domains. For simplicity, we shall from now on restrict our considerations to Poisson's equation; the results can be transformed to the general case (1.1).

Let $\Omega \subset \mathbf{R}^{2}$ be a bounded polygonal domain such that, for some sequence of uniform grids $\Omega_{h} \subset \Omega$ (with mesh size $h$ ), its boundary consists of horizontal, vertical or diagonal grid lines (see Figure 4.1). For this type of domains, explicit bounds are known for multigrid convergence rates; see Braess [5]. We are going to establish bounds for the contraction rate of the defect correction method.

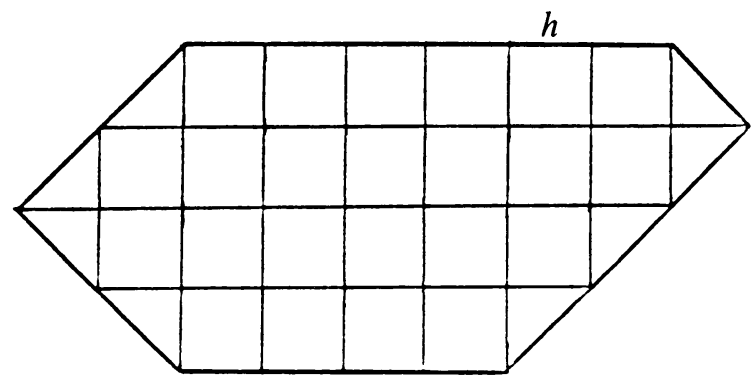

FIGURE 4.1

"Polygonal" domain

By $\partial \Omega_{h}$ we denote the intersection of $\partial \Omega$ with the grid lines. Let $\bar{\Omega}_{h}:=\Omega_{h}+\partial \Omega_{h}$. $P=(x, y) \in \bar{\Omega}_{h}$ is called (interior or boundary) grid point, respectively. $u_{h}: \bar{\Omega}_{h} \rightarrow \mathbf{R}$ is called grid function; often we shall tacitly extend a grid function to the infinite grid by $u_{h}(P):=0, P \notin \bar{\Omega}_{h}$. The linear space of grid functions is denoted by $\mathscr{U}_{h}$. $\mathscr{U}_{h}^{0}$ is the subspace of functions vanishing on $\partial \Omega_{h}$. Since the Dirichlet boundary condition will always be trivially satisfied, any "error function" henceforth considered is contained in $\mathscr{U}_{h}^{0}$.

Let $\Omega_{h} \subset \Omega_{h}$ be the set of grid points $P$ far enough away from the boundary such that the nine-point stencil (3.2) is well defined within $\bar{\Omega}_{h}$; let $\Gamma_{h}:=\Omega_{h} \backslash \Omega_{h}$. Define projection operators $R_{h}, B_{h}: \mathscr{U}_{h}^{0} \rightarrow \mathscr{U}_{h}^{0}$ by

$$
R_{h} u_{h}(P):= \begin{cases}u_{h}(P), & P \in \Omega_{h}, \\ 0, & P \in \Gamma_{h}\end{cases}
$$

and

$$
B_{h}:=I_{h}-R_{h}
$$


With respect to the discrete $L_{\infty}$-norm $\|\cdot\|_{\infty}$, the following estimate holds for the basic discretization $L_{h}$ (defined via (3.1)).

PROPOSITION 4.1.

$$
\left\|L_{h}^{-1} B_{h}\right\|_{\infty} \leqslant h^{2} \text {. }
$$

Proof. (4.2) is the reformulation of a "discrete Green's function estimate" in Bramble and Hubbard [6] (see also [1]).

Proposition 4.1 shows that the full order of consistency is not required near the boundary.

The target discretization $L_{h}^{\prime} u_{h}=f_{h}^{\prime}$ can be defined by the "Mehrstellenoperator" for $P \in \Omega_{h}$; for $P \in \Gamma_{h}$ we use the basic five-point scheme (3.1). Thus, the order of consistency is

$$
\left|\left(L_{h}^{\prime} u_{h}^{*}-f_{h}^{\prime}\right)(P)\right|= \begin{cases}O\left(h^{4}\right), & P \in \Omega_{h}, \\ O\left(h^{2}\right), & P \in \Gamma_{h} .\end{cases}
$$

$\Delta L_{h}=L_{h}-L_{h}^{\prime}$ is given by (3.2) for $P \in \Omega_{h}$; it is 0 for $P \in \Gamma_{h}$. Let $\mathscr{U}_{h}$ be equipped with the scalar product

$$
\left\langle u_{h}, v_{h}\right\rangle:=h^{2} \sum_{P \in \bar{\Omega}_{h}} u_{h}(P) v_{h}(P)
$$

Introducing discrete Sobolev norms, we shall write

$$
\left|u_{h}\right|_{H^{0}}:=\left\|u_{h}\right\|_{2}=\left\langle u_{h}, u_{h}\right\rangle^{1 / 2} \text {. }
$$

On $\mathscr{U}_{h}^{0}$, a $H^{1}$-norm can be defined by

$$
\left|u_{h}\right|_{H^{1}}:=\left[\left|\partial_{x} u_{h}\right|_{H^{0}}^{2}+\left|\partial_{y} u_{h}\right|_{H^{0}}^{2}\right]^{1 / 2}
$$

where $\partial x, \partial y$ are the first (forward-) difference quotients. (The corresponding backward-difference quotients will be denoted by $\bar{\partial} x, \bar{\partial} y$.) The dual of $|\cdot|_{H^{1}}$ is

$$
\left|u_{h}\right|_{H^{-1}}:=\sup _{0 \neq v_{h} \in \mathscr{U}_{h}^{0}} \frac{\left|\left\langle u_{h}, v_{h}\right\rangle\right|}{\left|v_{h}\right|_{H^{1}}} .
$$

For $u_{h} \in \mathscr{U}_{h}^{0}$ and $L_{h}$ as defined above, partial summation yields

$$
\left|u_{h}\right|_{H^{1}}=\left\langle L_{h} u_{h}, u_{h}\right\rangle^{1 / 2}=\left|L_{h}^{1 / 2} u_{h}\right|_{H^{0}} .
$$

(Note that $L_{h}$ is a symmetric, positive definite operator.) A discrete $H^{2}$-norm will be introduced later in this section. For the norm of an operator $A_{h}: \mathscr{U}_{h}^{0} \rightarrow \mathscr{U}_{h}$ we adopt the notation

$$
\left|A_{h}\right|_{s, t}:=\sup _{0 \neq u_{h} \in \mathscr{U}_{h}^{0}} \frac{\left|A_{h} u_{h}\right|_{H^{s}}}{\left|u_{h}\right|_{H^{t}}} .
$$

By virtue of the following lemma, the estimate (4.2) carries over to an estimate relative to $|\cdot|_{1,1}$ :

LEMMA 4.2.

$$
\left|L_{h}^{-1} B_{h}\right|_{1,1} \leqslant h^{2}
$$


Proof. (4.8) is equivalent to $\left|L_{h}^{-1}\right|_{1,-1}=1$. Hence it follows from (4.2) that

$$
\begin{aligned}
\left|L_{h}^{-1} B_{h}\right|_{1,1}^{2} & \leqslant\left|L_{h}^{-1}\right|_{1,-1}^{2}\left|B_{h}\right|_{-1,1}^{2} \\
& =\left|L_{h}^{-1 / 2} B_{h} L_{h}^{-1 / 2}\right|_{0,0}^{2}=\rho\left(L_{h}^{-1} B_{h} L_{h}^{-1} B_{h}\right) \\
& \leqslant\left\|L_{h}^{-1} B_{h} L_{h}^{-1} B_{h}\right\|_{\infty} \leqslant\left\|L_{h}^{-1} B_{h}\right\|_{\infty}^{2} \leqslant\left(h^{2}\right)^{2} .
\end{aligned}
$$

We shall need the following estimates for the projection operator $R_{h}: \mathscr{U}_{h}^{0} \rightarrow \mathscr{U}_{h}^{0}$.

LEMMA 4.3. We have

$$
\left|R_{h}\right|_{1,1} \leqslant \sqrt{7}
$$

If $\Omega$ is convex,

$$
\left|R_{h}\right|_{1,1} \leqslant \sqrt{2}
$$

Proof. (a) is established by the estimate

$$
\begin{aligned}
\left|R_{h}\right|_{1,1}^{2} & =\left|L_{h}^{1 / 2} R_{h} L_{h}^{-1 / 2}\right|_{0,0}^{2}=\rho\left(R_{h} L_{h}^{-1} R_{h} L_{h}\right) \\
& \leqslant\left\|R_{h}\right\|_{\infty}\left\|L_{h}^{-1} R_{h} L_{h}\right\|_{\infty} \leqslant 1+\left\|L_{h}^{-1} B_{h} L_{h}\right\|_{\infty} .
\end{aligned}
$$

It is easy to see that $\left\|B_{h} L_{h}\right\|_{\infty} \leqslant 6 h^{-2}$. Hence,

$$
\left|R_{h}\right|_{1,1} \leqslant\left(1+h^{2} 6 h^{-2}\right)^{1 / 2}=\sqrt{7}
$$

follows from Proposition 4.1.

For convex $\Omega$, the sharper bound (b) can be derived via direct estimates of the scalar products $\left\langle\partial_{x} R_{h} u_{h}, \partial_{x} R_{h} u_{h}\right\rangle$ and $\left\langle\partial_{y} R_{h} u_{h}, \partial_{y} R_{h} u_{h}\right\rangle$. See [2] for details.

Let $\bar{\Delta} L_{h}$ refer to the application of the Mehrstellenoperator (3.2) in every point of the infinite grid, irrespective of the boundary condition. In other words,

$$
\bar{\Delta} L_{h}:=\frac{h^{2}}{6} \bar{\partial}_{x} \partial_{x} \bar{\partial}_{y} \partial_{y} .
$$

Clearly, $\Delta L_{h}=R_{h} \bar{\Delta} L_{h}$.

LEMMA 4.4. We have

$$
\left|\bar{\Delta} L_{h}\right|_{-1,1} \leqslant \frac{1}{3} \quad \text { independent of } h .
$$

Proof. We consider the scalar product $\left\langle\bar{\Delta} L_{h} u_{h}, v_{h}\right\rangle$ and apply partial summation:

$$
\begin{aligned}
\left|\left\langle\bar{\Delta} L_{h} u_{h}, v_{h}\right\rangle\right| & =\frac{h^{2}}{12}\left[\left|\left\langle\bar{\partial}_{x} \partial_{x} \bar{\partial}_{y} \partial_{y} u_{h}, v_{h}\right\rangle\right|+\left|\left\langle\bar{\partial}_{x} \partial_{x} \bar{\partial}_{y} \partial_{y} u_{h}, v_{h}\right\rangle\right|\right] \\
& =\frac{h^{2}}{12}\left[\left|\left\langle\bar{\partial}_{y} \partial_{x} \partial_{y} u_{h}, \partial_{x} v_{h}\right\rangle\right|+\left|\left\langle\bar{\partial}_{x} \partial_{x} \partial_{y} u_{h}, \partial_{y} v_{h}\right\rangle\right|\right] \\
& \leqslant \frac{h^{2}}{12}\left|\partial_{x} \partial_{y} u_{h}\right|_{H^{1}}\left|v_{h}\right|_{H^{1}} \leqslant \frac{1}{3}\left|u_{h}\right|_{H^{1}}\left|v_{h}\right|_{H^{1}},
\end{aligned}
$$

where we have used the Cauchy-Schwarz inequality and crude estimation of $\left|\partial_{x} \partial_{y} u_{h}\right|_{H^{1}}$. Hence,

$$
\left|\bar{\Delta} L_{h} u_{h}\right|_{H^{-1}}=\sup _{\substack{0 \neq v_{h} \in \mathscr{U}_{h}^{0}\\}} \frac{\left|\left\langle\bar{\Delta} L_{h} u_{h}, v_{h}\right\rangle\right|}{\left|v_{h}\right|_{H^{1}}} \leqslant \frac{1}{3}\left|u_{h}\right|_{H^{1}} .
$$


THEOREM 4.5. With respect to $|\cdot|_{1,1}, L_{h}^{-1} \Delta L_{h}$ is a contraction:

$$
\left|L_{h}^{-1} \Delta L_{h}\right|_{1,1} \leqslant .882 \text {. }
$$

If $\Omega$ is convex,

$$
\left|L_{h}^{-1} \Delta L_{h}\right|_{1,1} \leqslant .472
$$

Proof. By (4.8),

$$
\begin{aligned}
& \left|L_{h}^{-1} \Delta L_{h} u_{h}\right|_{H^{1}} \leqslant\left|\Delta L u_{h}\right|_{H^{-1}}=\left|R_{h} \bar{\Delta} L_{h} u_{h}\right|_{H^{-1}} \\
& =\sup _{0 \neq v_{h} \in \mathscr{U}_{h}^{0}} \frac{\left|\left\langle\bar{\Delta} L_{h} u_{h}, R_{h} v_{h}\right\rangle\right|}{\left|v_{h}\right|_{H^{1}}} \leqslant \sup _{0 \neq v_{h} \in \mathscr{U}_{h}^{0}} \frac{\left|\bar{\Delta} L_{h} u_{h}\right|_{H^{-1}}\left|R_{h} v_{h}\right|_{H^{1}}}{\left|v_{h}\right|_{H^{1}}} \\
& \leqslant\left|R_{h}\right|_{1,1}\left|\bar{\Delta} L_{h}\right|_{-1,1}\left|u_{h}\right|_{H^{1}} \text {. }
\end{aligned}
$$

The result follows from Lemmas 4.3 and 4.4.

The analysis of the modified iteration (2.12) will require stronger properties of $L_{h}$ than used so far. We define $\partial_{x x}, \partial_{y y}: \mathscr{U}_{h}^{0} \rightarrow \mathscr{U}_{h}^{0}$ by

$$
\partial_{x x} u_{h}(P):= \begin{cases}\bar{\partial}_{x} \partial_{x} u_{h}(P), & P \in \Omega_{h}, \\ 0, & \text { else, }\end{cases}
$$

and similarly for $\partial_{y y}$. We introduce a discrete $H^{2}$-seminorm on $\mathscr{U}_{h}^{0}$ :

$$
\left|u_{h}\right|_{H^{2}}:=\left[\left|\partial_{x x} u_{h}\right|_{H^{0}}^{2}+\left|\partial_{y y} u_{h}\right|_{H^{0}}^{2}\right]^{1 / 2} \text {. }
$$

In the following theorem we present a quantitative discrete $H^{2}$-regularity estimate.

THEOREM 4.6. Discrete $H^{2}$-regularity. If $\Omega$ is convex,

$$
\left|u_{h}\right|_{H^{2}} \leqslant\left|L_{h} u_{h}\right|_{H^{0}}
$$

holds independently of $h$ for all $u_{h} \in \mathscr{U}_{h}^{0}$.

Proof. Let $u_{h} \in \mathscr{U}_{h}^{0}$. Since $L_{h}=-\left(\partial_{x x}+\partial_{y y}\right)$,

$$
\begin{aligned}
\left|u_{h}\right|_{H^{2}}^{2} & =\left\langle\partial_{x x} u_{h}, \partial_{x x} u_{h}\right\rangle+\left\langle\partial_{y y} u_{h}, \partial_{y y} u_{h}\right\rangle \\
& =\left\langle L_{h} u_{h}, L_{h} u_{h}\right\rangle-2\left\langle\partial_{x x} u_{h}, \partial_{y y} u_{h}\right\rangle \\
& =\left|L_{h} u_{h}\right|_{H^{0}}^{2}-2\left\langle\bar{\partial}_{x} \partial_{x} u_{h}, \partial_{y y} u_{h}\right\rangle .
\end{aligned}
$$

Now,

$$
\begin{aligned}
\left\langle\bar{\partial}_{x} \partial_{x} u_{h}, \partial_{y y} u_{h}\right\rangle & =\left\langle\bar{\partial}_{x} \partial_{x} u_{h}, \bar{\partial}_{y} \partial_{y} u_{h}\right\rangle+\left\langle\bar{\partial}_{x} \partial_{x} u_{h},\left(\partial_{y y}-\bar{\partial}_{y} \partial_{y}\right) u_{h}\right\rangle \\
& =\left\langle\partial_{x} \partial_{y} u_{h}, \partial_{x} \partial_{y} u_{h}\right\rangle-\left\langle\bar{\partial}_{x} \partial_{x} u_{h}, C_{h} \bar{\partial}_{y} \partial_{y} u_{h}\right\rangle .
\end{aligned}
$$

The first part is $\geqslant 0$, as required. In the second part, $C_{h}$ denotes restriction to the boundary points (because these are the only points where, in general, $\partial_{y y} u_{h}(P) \neq$ $\bar{\partial}_{y} \partial_{y} u_{h}(P)$ for $\left.u_{h} \in \mathscr{U}_{h}^{0}\right)$. We shall now investigate

$$
\gamma_{h}(P):=\bar{\partial}_{x} \partial_{x} u_{h}(P) \bar{\partial}_{y} \partial_{y} u_{h}(P)
$$

for all $P \in \partial \Omega_{h}$.

(a) $P$ lies on a horizontal or vertical grid line, but is not a corner point. Then $\gamma_{h}(P)=0$, since $\bar{\partial}_{x} \partial_{x} u_{h}(P)=0$ or $\bar{\partial}_{y} \partial_{y} u_{h}(P)=0$.

(b) $P$ is a corner point, the corner being not reentrant. Then again $\gamma_{h}(P)=0$, since either $\bar{\partial}_{x} \partial_{x} u_{h}(P)=0$ or $\bar{\partial}_{y} \partial_{y} u_{h}(P)=0$.

(a) and (b) imply $H^{2}$-regularity for rectangles. 


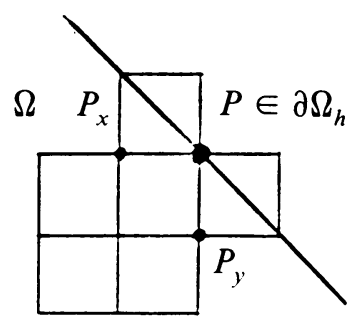

FIGURE 4.2

Boundary point on diagonal grid line

(c) $P$ lies on a diagonal grid line and is not a corner point. One of the possible situations is shown in Figure 4.2. Let $P_{x}:=P-h e_{x}, P_{y}:=P-h e_{y}$. Clearly, $\bar{\partial}_{x} \partial_{x} u_{h}(P)=h^{-2} u_{h}\left(P_{x}\right)$ and $\bar{\partial}_{y} \partial_{y} u_{h}(P)=h^{-2} u_{h}\left(P_{y}\right)$. The inequality $a b \leqslant \frac{1}{2}\left(a^{2}+b^{2}\right)$ yields

$$
\left|\gamma_{h}(P)\right| \leqslant \frac{1}{2}\left[\left(h^{-2} u_{h}\left(P_{x}\right)\right)^{2}+\left(h^{-2} u_{h}\left(P_{y}\right)\right)^{2}\right]
$$

Since $u_{h} \in \mathscr{U}_{h}^{0}$, we have

$$
\left|h^{-2} u_{h}\left(P_{x}\right)\right|=\left|\partial_{x} \partial_{y} u_{h}\left(P_{x}\right)\right|, \quad\left|h^{-2} u_{h}\left(P_{y}\right)\right|=\left|\partial_{x} \partial_{y} u_{h}\left(P_{y}\right)\right| .
$$

Similar conclusions hold for all other cases in question.

Since (a), (b) and (c) characterize arbitrary convex domains of the type under consideration, we have shown that, for convex $\Omega$,

$$
\begin{aligned}
\left|\left\langle\bar{\partial}_{x} \partial_{x} u_{h}, C_{h} \bar{\partial}_{y} \partial_{y} u_{h}\right\rangle\right| & \leqslant \sum_{P \in \partial \Omega_{h}}\left|\gamma_{h}(P)\right| \\
& \leqslant \frac{1}{2} \sum_{P \in \partial \Omega_{h}}\left[\left(\partial_{x} \partial_{y} u_{h}\left(P_{x}\right)\right)^{2}+\left(\partial_{x} \partial_{y} u_{h}\left(P_{y}\right)\right)^{2}\right],
\end{aligned}
$$

where it is sufficient to cover the points of type (c). It is obvious that, for $h$ not too large, the latter sum is bounded by $\left\langle\partial_{x} \partial_{y} u_{h}, \partial_{x} \partial_{y} u_{h}\right\rangle$, since no point $P_{x}, P_{y}$ will appear more than twice. Hence it follows from (4.18) that

$$
\left\langle\bar{\partial}_{x} \partial_{x} u_{h}, \partial_{y y} u_{h}\right\rangle \geqslant 0 \text {. }
$$

This establishes $H^{2}$-regularity in the convex case.

We shall from now on assume that $\Omega$ is convex. Theorem 4.6 enables us to establish a strengthened estimate for $L_{h}^{-1} \Delta L_{h}$.

LEMMA 4.7.

$$
\left|L_{h}^{-1} \Delta L_{h} L_{h}^{-1}\right|_{1,0} \leqslant .236 h .
$$

Proof. By (4.8) and Theorem 4.6 it suffices to show that

$$
\left|\Delta L_{h} u_{h}\right|_{H^{-1}} \leqslant .236 h\left|u_{h}\right|_{H^{2}}, \quad u_{h} \in \mathscr{U}_{h}^{0}
$$

Let $v_{h} \in \mathscr{U}_{h}^{0}$. Then,

$$
\begin{aligned}
\left|\left\langle\Delta L_{h} u_{h}, v_{h}\right\rangle\right| & =\left|\left\langle\Delta L_{h} u_{h}, R_{h} v_{h}\right\rangle\right| \\
& =\frac{h^{2}}{12}\left[\left|\left\langle\bar{\partial}_{x} \partial_{x} u_{h}, \bar{\partial}_{y} \partial_{y} R_{h} v_{h}\right\rangle\right|+\left|\left\langle\bar{\partial}_{y} \partial_{y} u_{h}, \bar{\partial}_{x} \partial_{x} R_{h} v_{h}\right\rangle\right|\right] \\
& =\frac{h^{2}}{12}\left[\left|\left\langle\partial_{x x} u_{h}, \bar{\partial}_{y} \partial_{y} R_{h} v_{h}\right\rangle\right|+\left|\left\langle\partial_{y y} u_{h}, \bar{\partial}_{x} \partial_{x} R_{h} v_{h}\right\rangle\right|\right],
\end{aligned}
$$


because the definition of $R_{h}$ implies that for any $P \in \partial \Omega_{h}$ either $\bar{\partial}_{x} \partial_{x} u_{h}(P)$ or $\bar{\partial}_{y} \partial_{y} R_{h} v_{h}(P)$ vanishes (similarly for the pair $\bar{\partial}_{y} \partial_{y}, \bar{\partial}_{x} \partial_{x} R_{h}$ ). Further partial summation and application of the Cauchy-Schwarz inequality yield

$$
\begin{aligned}
\left|\left\langle\Delta L_{h} u_{h}, v_{h}\right\rangle\right| & =\frac{h^{2}}{12}\left[\left|\left\langle\partial_{y} \partial_{x x} u_{h}, \partial_{y} R_{h} v_{h}\right\rangle\right|+\left|\left\langle\partial_{x} \partial_{y y} u_{h}, \partial_{x} R_{h} v_{h}\right\rangle\right|\right] \\
& \leqslant \frac{h^{2}}{12}\left[\left|\partial_{y} \partial_{x x} u_{h}\right|_{H^{0}}^{2}+\left|\partial_{x} \partial_{y y} u_{h}\right|_{H^{0}}^{2}\right]^{1 / 2}\left|R_{h}\right|_{1,1}\left|v_{h}\right|_{H^{1}}
\end{aligned}
$$

Hence, $\left|\Delta L_{h} u_{h}\right|_{H^{-1}}$ is bounded by

$$
\left|\Delta L_{h} u_{h}\right|_{H^{-1}} \leqslant \frac{h^{2}}{12}\left[\left(\frac{2}{h}\right)^{2}\left|u_{h}\right|_{H^{2}}^{2}\right]^{1 / 2} \sqrt{2}=\frac{2 \sqrt{2}}{12} h\left|u_{h}\right|_{H^{2}}<.236 h\left|u_{h}\right|_{H^{2}}
$$

by (4.11b) and crude estimates for $\partial_{x}, \partial_{y}$.

Consider now the modified defect correction iteration (2.12) involving a smoothing operator $S_{h}$ as described in Section 3. For $S_{h}$, the following smoothing property is required:

$$
\left|L_{h} S_{h}^{\nu}\right|_{0,1} \leqslant C(\nu) h^{-1}
$$

with $C(\nu) \rightarrow 0$ as $\nu \rightarrow \infty$.

Proposition 4.8. Let $S_{h}$ satisfy (4.21). Then,

$$
\left|L_{h}^{-1} \Delta L_{h} S_{h}^{\nu}\right|_{1,1} \leqslant 0.24 C(\nu) .
$$

Proof. Apply Lemma 4.7.

It follows from a result in Hackbusch [9, Section 3.3] that (4.21) is satisfied for Jacobi relaxation (with damping factor $\frac{1}{2}$ ) with

$$
C(\nu) \leqslant \frac{2 \sqrt{2}}{\sqrt{4 \nu-1}} \text {. }
$$

We also have

Proposition 4.9. For Gauss/Seidel relaxation with red-black ordering, (4.21) is satisfied with

$$
C(\nu) \leqslant \frac{2 \sqrt{2 \sqrt{2}}}{3 \sqrt{3 \sqrt{3} \nu}}
$$

Proof. See [2].

We summarize as follows.

Corollary 4.10. For convex $\Omega$,

$$
\left|L_{h}^{-1} \Delta L_{h}\right|_{1,1} \leqslant .472
$$

by Theorem 4.5. $\left|L_{h}^{-1} \Delta L_{h} S_{h}^{\nu}\right|_{1,1}$ is bounded by the numbers given in the following table:

\begin{tabular}{c||c|c}
$\nu$ & Jacobi & Gauss/Seidel \\
\hline \hline 1 & .386 & .349 \\
2 & .253 & .247 \\
3 & .202 & .202 \\
4 & .173 & .175
\end{tabular}


Our bounds are rigorous but certainly not optimal (cf. the model problem analysis in Section 3). The reason for this is that (4.19) as well as (4.21) are somewhat weaker than the corresponding estimates from Section 3. One of the open questions is whether something like $\left|L_{h}^{-1} \Delta L_{h} L_{h}^{-1}\right|_{1,1} \leqslant C h^{2}$ is satisfied.

Since our bounds are valid only in $H^{1}$, the investigation of the "fixed point shift" becomes more difficult than in Section 3. In particular, we get the following nonoptimal result. We note, in this connection, that Lemma 3.4 (applied to $|\cdot|_{0,0}$ ) carries over to the present case without modification.

Let $D_{h}: \mathscr{U}_{h}^{0} \rightarrow \mathscr{U}_{h}^{0}$ be defined by

$$
D_{h}:=R_{h}+h^{2} B_{h} \text {. }
$$

Proposition 4.11. Let $\left|L_{h}^{-1} \Delta L_{h} S_{h}^{\nu}\right|_{1,1} \leqslant m<1$ (cf. Corollary 4.10). There exist constants $C_{1}, C_{2}$ independent of $h$ such that the error $\hat{e}_{h}$ of Proposition 2.2 satisfies

$$
\left|\hat{e}_{h}\right|_{H^{1}} \leqslant \frac{1}{h} \frac{1}{1-m}\left[C_{1}\left|D_{h}\left(L_{h}^{\prime} u_{h}^{*}-f_{h}^{\prime}\right)\right|_{H^{0}}+C_{2} C^{\prime}(\nu) h^{2}\left|L_{h} u_{h}^{*}-f_{h}\right|_{H^{0}}\right]
$$

(with $C^{\prime}(\nu)$ from Lemma 3.4). Thus, $\left|\hat{e}_{h}\right|_{H^{1}}=O\left(h^{3}\right)$.

Proof. By construction, the truncation error of the target discretization is $O\left(h^{4}\right)$ in the interior points of $P \in \Omega_{h}$ and $O\left(h^{2}\right)$ for $P \in \Gamma_{h}$. Therefore,

$$
\left|D_{h}\left(L_{h}^{\prime} u_{h}^{*}-f_{h}^{\prime}\right)\right|_{H^{0}} \leqslant C h^{4} \text {. }
$$

By (4.10), $\left|L_{h}^{-1} D_{h}^{-1}\right|_{1,1} \leqslant C$. Hence it follows from Lemmas 3.4 and 4.7 that

$$
\begin{aligned}
\left|\hat{e}_{h}\right|_{H^{1}} \leqslant & \frac{1}{1-m}\left[\left|L_{h}^{-1} D_{h}^{-1}\right|_{1,1}\left|D_{h}\left(L_{h}^{\prime} u_{h}^{*}-f_{h}^{\prime}\right)\right|_{H^{1}}\right. \\
& \left.\quad+\left|L_{h}^{-1} \Delta L_{h} L_{h}^{-1}\right|_{1,0}\left|L_{h}\right|_{0,0}\left|T_{h}\right|_{0,0}\left|L_{h} u_{h}^{*}-f_{h}\right|_{H^{0}}\right] \\
\leqslant & \frac{1}{1-m}\left[C_{1} h^{-1}\left|D_{h}\left(L_{h}^{\prime} u_{h}^{*}-f_{h}^{\prime}\right)\right|_{H^{0}}+.236 h C h^{-2} C^{\prime}(\nu) h^{2} h^{2}\right] \\
= & O\left(h^{3}\right) .
\end{aligned}
$$

We have shown $O\left(h^{3}\right)$ for $\left|\hat{e}_{h}\right|_{H^{0}}$ and for $\left|\partial_{x} \hat{e}_{h}\right|_{H^{0}},\left|\partial_{y} \hat{e}_{h}\right|_{H^{0}}$. It is easy to see that $O\left(h^{4}\right)$ follows if the truncation errors can be measured in $H^{1}$ rather than $H^{0}$ without loss of order. If $u^{*}$ is sufficiently smooth, this can be expected in an "interior sense", but not up to the boundary.

On the other hand, estimates for $\left|L_{h}^{-1} \Delta L_{h}\right|_{0,0}$ seem to be very hard to obtain. In particular, numerical experience tells us that $\left|L_{h}^{-1} \Delta L_{h}\right|_{0,0}<1$ cannot be expected in general. We have found an example where $\left|L_{h}^{-1} \Delta L_{h}\right|_{0,0}>1$, even though $\Omega$ is convex (cf. [1]). (In contrast to this, $\left|\Delta L_{h} L_{h}^{-1}\right|_{0,0}<1$ can easily be derived from Theorem 4.6.)

5. General Domains; Concluding Remarks. On the basis of the work of Hackbusch [8], [11], much of the reasoning from Section 4 can (at least qualitatively) be extended to the case of (1.1) in a domain $\Omega$ with curved boundary. The remarks below contain a summary of our analysis for general domains, which can be found in [2].

Suitable (basic and target) discretizations of (1.1) involve special difference formulae for "irregular" points near the boundary (see [2] for details). For the usual "Shortley-Weller"-operator $L_{h}$, Proposition 4.1 remains valid without modification 
(cf. [6]). The discrete regularity properties of the Shortley-Weller scheme have been studied by Hackbusch [11] (see also [1]). For discrete $H^{1}$ - and $H^{2}$-norms appropriately defined, the following estimates hold independently of the mesh size $h$ :

$$
\left|L_{h}^{-1}\right|_{1,-1} \leqslant 1.02
$$

is valid under very weak assumptions on $\Omega$, whereas

$$
\left|L_{h}^{-1}\right|_{2,0} \leqslant C
$$

is only true if the boundary $\partial \Omega$ is sufficiently smooth. At present, the constant in (5.2) is not explicitly known.

Using (5.1), (5.2) and analogues of Lemmas 4.2-4.4, it is shown in [2] that $\left|L_{h}^{-1} \Delta L_{h}\right|_{1,1}$ is $O(1)$. A further result is

$$
\left|L_{h}^{-1} \Delta L_{h} L_{h}^{-1}\right|_{1, \theta-1}=O\left(h^{\theta}\right), \quad 0 \leqslant \theta<\frac{1}{2}
$$

where $|\cdot|_{H^{\theta}}$ is a discrete Sobolev norm of noninteger order. Combining (5.3) with a generalized smoothing property, which is proved in Hackbusch [8] for Jacobi and Gauss/Seidel smoothers $S_{h}$, we obtain

$$
\left|L_{h}^{-1} \Delta L_{h} S_{h}^{\nu}\right|_{1,1} \leqslant C(\nu)
$$

where $C(\nu) \rightarrow 0$ for $\nu \rightarrow \infty$. Thus, the defect correction is contractive if a sufficient ( $h$-independent) number of smoothing sweeps are performed.

The defect correction algorithm described in this paper has been implemented on the basis of the standard multigrid solver MG01 for Helmholtz's equation (cf. Stüben and Trottenberg [13]). A detailed description is given in [3]. Numerical experiences are reported in [1] and [3].

Our final remark deals with the question of stability of the target discretization. On the one hand, stability of $L_{h}^{\prime}$ is a direct consequence of $\rho\left(L_{h}^{-1} \Delta L_{h}\right)<1$, independently of $h$ (cf. Sections 3 and 4). However, Proposition 2.2 shows how, in principle, error smoothing may help when using an unstable $L_{h}^{\prime}$. This could be of particular interest for such types of problems where high-order schemes inevitably are unstable (nonelliptic or singularly perturbed problems as, e.g., the convection diffusion equation). Future work will be concerned with this subject.

Institut für Angewandte und Numerische Mathematik

Technische Universität Wien

Wiedner Hauptstrasse 6-10

A-1040 Wien, Austria

1. W. AuZINGER, Defektkorrektur für Diskretisierungen des Dirichlet-Problems in allgemeinen Gebieten, Ph. D. Thesis, Technical University of Vienna, Oct., 1984.

2. W. Auzinger, Defect Corrections for Multigrid Solutions of the Dirichlet Problem in General Domains, Report 61/85, Inst. f. Angewandte und Numerische Mathematik, Technical University of Vienna, 1985.

3. W. Auzinger, DCMG01: A Multigrid Code with Defect Correction to Solve $\Delta U-c(x, y) U=$ $f(x, y)$ (on $\Omega), U=g(x, y)$ (on $\partial \Omega$ ), on Nonrectangular Bounded Domains $\Omega$ with High Accuracy, Arbeitspapier der GMD, Nr. 127, St. Augustin, Germany, January, 1985.

4. W. Auzinger \& H. J. SteTter, Defect Corrections and Multigrid Iterations, in Lecture Notes in Math., vol. 960, Springer-Verlag, Berlin and New York, 1982, pp. 327-351.

5. D. BRAESS, "The convergence rate of a multigrid method with Gauss-Seidel relaxation for the Poisson equation," Math. Comp., v. 42, 1984, pp. 505-519.

6. J. H. Bramble \& B. E. Hubbard, "On the formulation of finite difference analogues of the Dirichlet problem for Poisson's equation,” Numer. Math., v. 4, 1962, pp. 313-327. 
7. R. Frank, J. Hertling \& J. P. Monnet, "The application of iterated defect correction to variational methods for elliptic boundary value problems," Computing, v. 30, 1983, pp. 121-135.

8. W. HACKBUSCH, "Convergence of multi-grid iterations applied to difference equations," Math. Comp., v. 34, 1980, pp. 425-440.

9. W. HaCKBUSCH, Multigrid Convergence Theory, in Lecture Notes in Math., vol. 960, Springer-Verlag, Berlin and New York, 1982, pp. 177-219.

10. W. HaCKBuSCH, On Multigrid Iterations with Defect Corrections, in Lecture Notes in Math., vol. 960, Springer-Verlag, Berlin and New York, 1982, pp. 461-473.

11. W. HaCkBUSCH, “On the regularity of difference schemes. Part II: Regularity estimates for linear and nonlinear problems," Ark. Mat., v. 21, 1983, pp. 1-28.

12. H. J. STETtER, "The defect correction principle and discretization methods," Numer. Math., v. 29 , 1978, pp. 425-443.

13. K. Stüben \& U. Trottenberg, Multigrid Methods: Fundamental Algorithms, Model Problem Analysis and Applications, in Lecture Notes in Math., vol. 960, Springer-Verlag, Berlin and New York, 1982, pp. 1-176. 